\title{
A KINEMATIC VARIATIONAL PRINCIPLE FOR THIN METALLIC AND COMPOSITE PLATES EXPERIENCING LARGE DEFLECTIONS ABOVE THE VON KARMAN LIMITS
}

\author{
Sergey Selyugin \\ Stade, Germany \\ e-mail: sergey.selyugin@t-online.de
}

Thin elastic plates (metallic or composite) experiencing large deflections are considered. The plate deflections are much larger than the plate thickness. The geometrically nonlinear elasticity theory and the Kirchhoff assumptions are employed. The elongations, the shears and the in-plane rotations are assumed to be small. A kinematic variational principle leading to a boundary value problem for the plate is derived. It is shown that the principle gives proper equilibrium equations and boundary conditions. For moderate plate deflections the principle is transformed to the case of the von Karman plate.

Keywords: thin plate, metal, composite, large deflection, geometric nonlinearity, kinematic variational principle

(C) Sergey Selyugin, 2021 


\section{Introduction}

As the thin-walled structures are widely used now, many researchers explore the nonlinear behavior of thin elastic flexible plates. In case of the Kirchhoff assumptions the theory described in (Washizu 1975, Reddy 2007) is used. The theory employs the von Karman approach.

It is known that the latter approach describes the so-called moderate deflections (see Novozhilov 1961, 2011). The deflections are supposed to be comparable to the plate thickness. In (Reddy 2007) an opinion is indicated that in the von Karman approach the plate out-of-plane rotations should not be greater than $10^{\circ}-15^{\circ}$.

On the other hand, there are cases of considerable or even large deflections (much larger than the plate thickness); the cases need to be explored also.

The present paper deals with the case of large deflections. The equilibrium equations and the boundary conditions for the case have been obtained in (Selyugin 2021). The deflections created due to the static loading are much larger than the plate thickness. A kinematic variational principle leading to the equations and to the proper boundary conditions is derived.

\section{Derivation of a kinematic variational principle}

\subsection{Assumptions}

We consider a thin linear-elastic plate (metallic or composite) of constant thickness $h$. The plate thickness is much smaller than the linear plate dimensions. Fig. 1 demonstrates the coordinate system and some used notations. The X-Y plane coincides with the mid-plane $\Gamma$ of the plate. The plate is loaded in $-Z$ direction by the pressure $q$. The plate lateral surface $S$ consists of the statically loaded part $S_{1}$ (the X, Y, Z forces per unit area are $f_{x}, f_{y}, f_{z}$, respectively) and the part with the prescribed displacements $S_{2}$. The mid-plane $\Gamma$ intersects the lateral surface $S$ at the smooth contour $C$, with the portion $C_{1}$ belonging to $S_{1}$ and the portion $C_{2}$ belonging to $S_{2}$. The normal to $C$ is the vector $\vec{n}$ with the components $l, m$. The tangential direction to $C$ is $s$. The $n, s, z$ directions create the right triplet of vectors. The points of non-smoothness at the contour $C$ will be considered in a separate paper in a way similar to the one of (Washizu 1975).

The plate experiences large deflections generated due to static loading (they are much larger than the plate thickness).

The geometrically nonlinear elasticity theory and the Kirchhoff assumptions are employed (Novozhilov 1961, 2011). The latter assumptions are written as follows:

$$
\varepsilon_{z z}=\varepsilon_{x z}=\varepsilon_{y z}=0
$$

where $\varepsilon$ is the Green strain tensor.

The deformed plate is assumed to be stable.

In this sub-Section we repeat some relations from (Selyugin 2021) for having all necessary items in one location. 


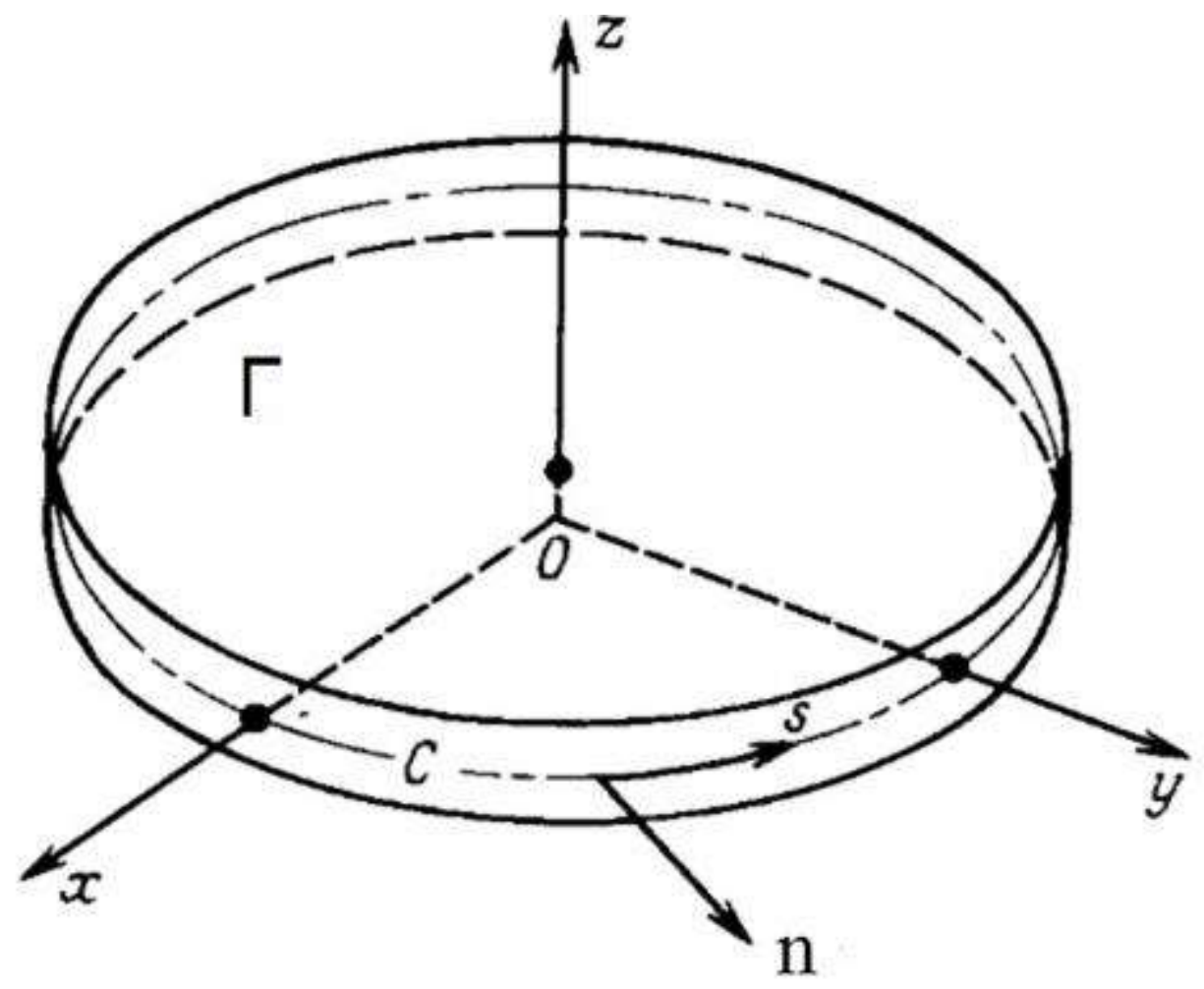

Fig. 1.

The ordinary notations are used hereinafter

$$
\begin{aligned}
& \frac{\partial}{\partial x}(\ldots)=(\ldots),{ }_{x} \\
& \frac{\partial}{\partial y}(\ldots)=(\ldots),{ }_{y}
\end{aligned}
$$

Small elongations, small shears and small squared in-plane rotations (negligible as compared to unity) are assumed. Following (Novozhilov, 1961, 2011), the displacements $u, v, w$ within the plate (respectively along $\mathrm{X}, \mathrm{Y}, \mathrm{Z}$ ) are supposed to be

$$
\begin{aligned}
& u(x, y)=\hat{u}(x, y)+z \cdot \theta(x, y) \\
& \mathrm{v}(x, y)=\hat{\mathrm{v}}(x, y)+z \cdot \psi(x, y) \\
& w(x, y)=\hat{w}(x, y)+z \cdot \chi(x, y)
\end{aligned}
$$

where upper 'tilde' means quantities at the mid-plane.

After using (3) and the definition of the Green tensor components it is obtained in (Novozhilov, 1961, 2011) that

$$
\begin{aligned}
& \theta=-\hat{w},_{x}\left(1+\hat{\mathrm{v}},{ }_{y}\right)+\hat{\mathrm{v}},,_{x} \hat{w},{ }_{y} \\
& \psi=-\hat{w},{ }_{y}\left(1+\hat{u},{ }_{x}\right)+\hat{u},{ }_{y} \hat{w},{ }_{x} \\
& \chi=\hat{u},{ }_{x}+\hat{\mathrm{v}},{ }_{y}+\hat{u},{ }_{x} \hat{\mathrm{v}},{ }_{y}-\hat{u},{ }_{y} \hat{\mathrm{v}},{ }_{x}
\end{aligned}
$$

and 


$$
\begin{aligned}
& \varepsilon_{x x}=\hat{\varepsilon}_{x x}+z \kappa_{x x}+z^{2} \eta_{x x} \\
& \varepsilon_{y y}=\hat{\varepsilon}_{y y}+z \kappa_{y y}+z^{2} \eta_{y y} \\
& \varepsilon_{x y}=\hat{\varepsilon}_{x y}+z \kappa_{x y}+z^{2} \eta_{x y}
\end{aligned}
$$

The mid-plane strains in (5) are

$$
\begin{aligned}
& \hat{\varepsilon}_{x x}=\hat{u},{ }_{x}+1 / 2\left[\hat{u}_{x}{ }_{x}+\hat{\mathrm{v}},{ }_{x}{ }_{x}+\hat{w}_{x_{x}}{ }^{2}\right] \\
& \hat{\varepsilon}_{y y}=\hat{\mathrm{v}},_{y}+1 / 2\left[\hat{u},{ }_{y}^{2}+\hat{\mathrm{v}}_{y}^{2}+\hat{w},{ }_{y}^{2}\right] \\
& 2 \hat{\varepsilon}_{x y}=\hat{u},_{y}+\hat{\mathrm{v}},_{x}+\hat{u},{ }_{y} \hat{u},{ }_{x}+\hat{\mathrm{v}},{ }_{x} \hat{\mathrm{v}},{ }_{y}+\hat{w},{ }_{x} \hat{w}_{y}
\end{aligned}
$$

and other quantities in (5):

$$
\begin{aligned}
& \kappa_{x x}=\theta,_{x}+\hat{u},{ }_{x} \theta,,_{x}+\hat{\mathrm{v}},{ }_{x} \psi,{ }_{x}+\hat{w},{ }_{x} \chi,_{x} \\
& \kappa_{y y}=\psi,{ }_{y}+\hat{u},{ }_{y} \theta,{ }_{y}+\hat{\mathrm{v}},{ }_{y} \psi,{ }_{y}+\hat{w},{ }_{y} \chi,_{y} \\
& 2 \kappa_{x y}=\theta,_{y}+\psi,,_{x}+\hat{u},{ }_{x} \theta,_{y}+\hat{u},{ }_{y} \theta,,_{x}+\hat{\mathrm{v}},{ }_{x} \psi,{ }_{y}+\hat{\mathrm{v}},{ }_{y} \psi,{ }_{x}+\hat{w},{ }_{x} \chi,_{y}+\hat{w},{ }_{y} \chi,_{x} \\
& \eta_{x x}=1 / 2\left[\theta,{ }_{x}^{2}+\psi,{ }_{x}^{2}+\chi,{ }_{x}^{2}\right] \\
& \eta_{y y}=1 / 2\left[\theta,{ }_{y}^{2}+\psi,{ }_{y}^{2}+\chi,{ }_{y}^{2}\right] \\
& 2 \eta_{x y}=\theta,{ }_{x} \theta,{ }_{y}+\psi,{ }_{x} \psi,{ }_{y}+\chi,{ }_{x} \chi,{ }_{y}
\end{aligned}
$$

In (Novozhilov, 1961, 2011) it is indicated that for small elongations and shears the last (quadratic wrt to $z$ ) terms in (5) are small enough and may be neglected. Then (5) is transformed into

$$
\begin{aligned}
& \varepsilon_{x x}=\hat{\varepsilon}_{x x}+z \kappa_{x x} \\
& \varepsilon_{y y}=\hat{\varepsilon}_{y y}+z \kappa_{y y} \\
& \varepsilon_{x y}=\hat{\varepsilon}_{x y}+z \kappa_{x y}
\end{aligned}
$$

Substituting (4) in (7) and keeping in mind the small in-plane rotations, after cumbersome transformations we obtain (see Selyugin 2021)

$$
|\chi|<<1
$$

and

$$
\begin{aligned}
& \kappa_{x x}=-\theta(x, y) \hat{u},_{x x}-\psi(x, y) \hat{\mathrm{v}},_{x x}-\hat{w},_{x x} \\
& \kappa_{y y}=-\theta(x, y) \hat{u},_{y y}-\psi(x, y) \hat{\mathrm{v}},_{y y}-\hat{w},_{y y} \\
& \kappa_{x y}=-\theta(x, y) \hat{u},_{x y}-\psi(x, y) \hat{\mathrm{v}},_{x y}-\hat{w},_{x y}
\end{aligned}
$$

\subsection{Generalized stress-strain relations}

The equilibrium equations derived in (Selyugin 2021) require the generalized stress-strain relations, namely, the relations of the in-plane forces and of the bending/twisting moments as functions of the mid-plane strains (6) and of the quantities $\kappa_{x x}, \kappa_{y y}, \kappa_{x y}(11)$. 
For obeying the Hook's law metallic plates the $A$ and $D$ stiffness matrices, introduced analogous to the composite plate theory (Gibson 1994), are given by

$$
A=h\left[\begin{array}{ccc}
\frac{E}{1-\mu^{2}} & \frac{E \mu}{1-\mu^{2}} & 0 \\
\frac{E \mu}{1-\mu^{2}} & \frac{E}{1-\mu^{2}} & 0 \\
0 & 0 & \frac{E}{2(1+\mu)}
\end{array}\right] ; \quad D=\frac{h^{3}}{12}\left[\begin{array}{ccc}
\frac{E}{1-\mu^{2}} & \frac{E \mu}{1-\mu^{2}} & 0 \\
\frac{E \mu}{1-\mu^{2}} & \frac{E}{1-\mu^{2}} & 0 \\
0 & 0 & \frac{E}{2(1+\mu)}
\end{array}\right]
$$

where $E, \mu$ are the Young module and the Poisson ratio, respectively.

The matrices are used in the relations coupling the vectors of the in-plane forces and the bending/twisting moments (per unit length) $\vec{N}, \vec{M}$ and $\vec{\varepsilon}, \vec{\kappa}$.

We introduce the following notations

$$
\begin{aligned}
& N_{x}=\int_{-h / 2}^{h / 2} \sigma_{x x} d z, \quad M_{x}=\int_{-h / 2}^{h / 2} z \sigma_{x x} d z, \quad F_{x}=\int_{-h / 2}^{h / 2} f_{x} d z, \quad \tilde{M}_{x}=\int_{-h / 2}^{h / 2} z f_{x} d z \\
& N_{y}=\int_{-h / 2}^{h / 2} \sigma_{y y} d z, \quad M_{y}=\int_{-h / 2}^{h / 2} z \sigma_{y y} d z, \quad F_{y}=\int_{-h / 2}^{h / 2} f_{y} d z, \quad \tilde{M}_{y}=\int_{-h / 2}^{h / 2} z f_{y} d z \\
& N_{x y}=\int_{-h / 2}^{h / 2} \sigma_{x y} d z, \quad M_{x y}=\int_{-h / 2}^{h / 2} z \sigma_{x y} d z \quad F_{z}=\int_{-h / 2}^{h / 2} f_{z} d z \quad \widetilde{F}_{z}=\int_{-h / 2}^{h / 2} z f_{z} d z \\
& \vec{N}=\left(\begin{array}{c}
N_{x} \\
N_{y} \\
N_{x y}
\end{array}\right) \quad ; \quad \vec{M}=\left(\begin{array}{c}
M_{x} \\
M_{y} \\
M_{x y}
\end{array}\right) \\
& \vec{N}=A \vec{\varepsilon} \quad ; \quad \vec{M}=D \vec{\kappa}
\end{aligned}
$$

where $\vec{\kappa}$ is a vector characterizing the plate mid-surface curvature in case of large deflections (the vector is given below). The vector $\vec{\varepsilon}$ is composed of the in-plane strain components,

$$
\begin{aligned}
& \vec{\kappa}=\left(\begin{array}{c}
\kappa_{x x} \\
\kappa_{y y} \\
2 \kappa_{x y}
\end{array}\right) \\
& \vec{\varepsilon}=\left(\begin{array}{c}
\varepsilon_{x x} \\
\varepsilon_{y y} \\
2 \varepsilon_{x y}
\end{array}\right)
\end{aligned}
$$


For composite plates (in the present paper their lay-up is supposed to be symmetric) the $A$ and $D$ stiffness matrices are well-described in numerous composite textbooks like (Gibson 1994). A generalization to the non-symmetric lay-up case is straightforward and will not be presented here.

\subsection{Strain potential energy and its variation} (1)):

The strain potential energy $\Pi$ of the plate is given by the following formula (remembering

$$
\Pi=\int_{V} \frac{1}{2} \sigma_{i j} \varepsilon_{i j} d V=\int_{\Gamma}\left[\frac{1}{2}(\vec{N}, \hat{\vec{\varepsilon}})+\frac{1}{2}(\vec{M}, \vec{\kappa})\right] d \Gamma=\int_{\Gamma}\left[\frac{1}{2}\left(\hat{\vec{\varepsilon}}^{T} A \hat{\vec{\varepsilon}}\right)+\frac{1}{2}\left(\vec{\kappa}^{T} D \vec{\kappa}\right)\right] d \Gamma
$$

where $\sigma_{i j}$ is the second Piola-Kirchhoff stress tensor, $T$ means transposing, the repeated subscripts mean summation, two vectors in round brackets with comma between the vectors mean the scalar product of the vectors.

Now we perform the variation of $\Pi$ wrt the mid-surface displacements $\hat{u}, \hat{v}, \hat{w}$ :

$\delta \Pi=\int_{\Gamma}[(\vec{N}, \delta \hat{\vec{\varepsilon}})+(\vec{M}, \delta \vec{\kappa})] d \Gamma=\int_{\Gamma}\left[N_{x} \delta \varepsilon_{x x}+N_{y} \delta \varepsilon_{y y}+2 N_{x y} \delta \varepsilon_{x y}+M_{x} \delta \kappa_{x x}+M_{y} \delta \kappa_{y y}+2 M_{x y} \delta \kappa_{x y}\right] d \Gamma$

where (14)-(15) are used for the forces and moments. We have six terms under the rhs integral in (19). They are calculated one by one below. Some cumbersome transformations are omitted.

$$
\begin{aligned}
& N_{x} \delta \varepsilon_{x x}=N_{x} \delta\left[\hat{u},,_{x}+\frac{1}{2}\left(\hat{u},{ }_{x}^{2}+\hat{v},{ }_{x}^{2}+\hat{w},{ }_{x}^{2}\right)\right]=\frac{\partial}{\partial x}\left[N_{x} \delta \hat{u}+N_{x} \hat{u},_{x} \delta \hat{u}+N_{x} \hat{v},_{x} \delta \hat{v}+N_{x} \hat{w},{ }_{x} \delta \hat{w}\right]- \\
& -N_{x, x} \delta \hat{u}-\left(N_{x} \hat{u},{ }_{x}\right){ }_{x} \delta \hat{u}-\left(N_{x} \hat{v},{ }_{x}\right){ }_{x} \delta \hat{v}-\left(N_{x} \hat{w},{ }_{x}\right){ }_{x} \delta \hat{w} \\
& N_{y} \delta \varepsilon_{y y}=\delta\left[\hat{v},_{y}+\frac{1}{2}\left(\hat{u},{ }_{y}^{2}+\hat{v}_{,}^{2}+\hat{w},{ }_{y}^{2}\right)\right]=\frac{\partial}{\partial y}\left[N_{y} \delta \hat{v}+N_{y} \hat{u},{ }_{y} \delta \hat{u}+N_{y} \hat{v},{ }_{y} \delta \hat{v}+N_{y} \hat{w},{ }_{y} \delta \hat{w}\right]- \\
& -N_{y, y} \delta \hat{v}-\left(N_{y} \hat{u},{ }_{y}\right),{ }_{y} \delta \hat{u}-\left(N_{y} \hat{v},{ }_{y}\right), y \hat{v}-\left(N_{y} \hat{w},{ }_{y}\right), y \\
& 2 N_{x y} \delta \varepsilon_{x y}=2 N_{x y} \delta\left[\frac{1}{2}\left(\hat{u},{ }_{y}+\hat{v},_{x}\right)+\frac{1}{2}\left(\hat{u},{ }_{y} \hat{u},_{x}+\hat{v},{ }_{y} \hat{v}_{x}+\hat{w},{ }_{y} \hat{w},_{x}\right)\right]= \\
& =\frac{\partial}{\partial x}\left[N_{x y} \delta \hat{v}+N_{x y} \hat{u},{ }_{y} \delta \hat{u}+N_{x y} \hat{v},_{y} \delta \hat{v}+N_{x y} \hat{w},{ }_{y} \delta \hat{w}\right]+ \\
& +\frac{\partial}{\partial y}\left[N_{x y} \delta \hat{u}+N_{x y} \hat{u},_{x} \delta \hat{u}+N_{x y} \hat{v},_{x} \delta \hat{v}+N_{x y} \hat{w},_{x} \delta \hat{w}\right]- \\
& -N_{x y, x} \delta \hat{v}-\left(N_{x y} \hat{u},{ }_{y}\right),_{x} \delta \hat{u}-\left(N_{x y} \hat{v},_{y}\right){ }_{x} \delta \hat{v}-\left(N_{x y} \hat{w},{ }_{y}\right){ }_{x} \delta \hat{w}- \\
& -N_{x y, y} \delta \hat{u}-\left(N_{x y} \hat{u},_{x}\right),{ }_{y} \delta \hat{u}-\left(N_{x y} \hat{v}_{x}\right),_{y} \delta \hat{v}-\left(N_{x y} \hat{w},_{x}\right),_{y} \delta \hat{w}
\end{aligned}
$$




$$
\begin{aligned}
& M_{x} \delta \kappa_{x x}=M_{x}\left[-\theta \delta \hat{u},{ }_{x x}-\hat{u},_{x x} \delta \theta-\psi \delta \hat{v},_{x x}-\hat{v},_{x x} \delta \psi-\delta \hat{w},_{x x}\right]= \\
& =\frac{\partial}{\partial x}\left[-M_{x} \theta \delta \hat{u},{ }_{x}-\left(-M_{x} \theta\right),{ }_{x} \delta \hat{u}-M_{x} \psi \delta \hat{v},{ }_{x}-\left(-M_{x} \psi\right),{ }_{x} \delta \hat{v}-M_{x} \delta \hat{w},_{x}-\left(-M_{x}\right),_{x} \delta \hat{w}\right]- \\
& -\left(M_{x} \theta\right),_{x x} \delta \hat{u}-M_{x} \hat{u},_{x x} \delta \theta-\left(M_{x} \psi\right),_{x x} \delta \hat{v}-M_{x} \hat{v},_{x x} \delta \psi-\left(M_{x}\right),_{x x} \delta \hat{w} \\
& M_{y} \delta \kappa_{y y}=M_{y}\left\lfloor-\theta \delta \hat{u},_{y y}-\hat{u},_{y y} \delta \theta-\psi \delta \hat{v},_{y y}-\hat{v},_{y y} \delta \psi-\delta \hat{w},_{y y}\right\rfloor= \\
& =\frac{\partial}{\partial y}\left[-M_{y} \theta \delta \hat{u},{ }_{y}-\left(-M_{y} \theta\right),{ }_{y} \delta \hat{u}-M_{y} \psi \delta \hat{v},{ }_{y}-\left(-M_{y} \psi\right),{ }_{y} \delta \hat{v}-M_{y} \delta \hat{w},{ }_{y}-\left(-M_{y}\right),_{y} \delta \hat{w}\right]- \\
& -\left(M_{y} \theta\right),_{y y} \delta \hat{u}-M_{y} \hat{u},_{y y} \delta \theta-\left(M_{y} \psi\right), y y=\hat{v}-M_{y} \hat{v},_{y y} \delta \psi-\left(M_{y}\right),{ }_{y y} \delta \hat{w} \\
& 2 M_{x y} \delta \kappa_{x y}=2 M_{x y}\left\lfloor-\theta \delta \hat{u},_{x y}-\hat{u},_{x y} \delta \theta-\psi \delta \hat{v},_{x y}-\hat{v},_{x y} \delta \psi-\delta \hat{w},_{x y}\right\rfloor= \\
& =\frac{\partial}{\partial x}\left[-M_{x y} \theta \delta \hat{u},{ }_{y}-\left(-M_{x y} \theta\right), y \hat{u}-M_{x y} \psi \delta \hat{v},{ }_{y}-\left(-M_{x y} \psi\right),{ }_{y} \delta \hat{v}-M_{x y} \delta \hat{w},_{y}-\left(-M_{x y}\right), y \hat{w}\right]+ \\
& +\frac{\partial}{\partial y}\left[-M_{x y} \theta \delta \hat{u},{ }_{x}-\left(-M_{x y} \theta\right),_{x} \delta \hat{u}-M_{x y} \psi \delta \hat{v},_{x}-\left(-M_{x y} \psi\right),_{x} \delta \hat{v}-M_{x y} \delta \hat{w},{ }_{x}-\left(-M_{x y}\right),_{x} \delta \hat{w}\right] \\
& \left.\left.-\left(2 M_{x y} \theta\right)\right)_{x y} \delta \hat{u}-2 M_{x y} \hat{u},_{x y} \delta \theta-\left(2 M_{x y} \psi\right)\right)_{x y} \delta \hat{v}-2 M_{x y} \hat{v},_{x y} \delta \psi-\left(2 M_{x y}\right),_{x y} \delta \hat{w}
\end{aligned}
$$

In (25) it is taken into account, that for two functions $a$ and $b$ of $(x, y)$ the following relation is valid:

$$
a b,_{x y}=\frac{1}{2} \frac{\partial}{\partial x}\left(a b,,_{y}-a,,_{y} b\right)+\frac{1}{2} \frac{\partial}{\partial y}\left(a b,,_{x}-a,{ }_{x} b\right)+a,,_{x y} b
$$

Introducing two functions of $(x, y)$

$$
\begin{aligned}
& \Omega=-M_{x} \hat{v}_{x x}-M_{y} \hat{v},_{y y}-2 M_{x y} \hat{v},_{x y} \\
& R=-M_{x} \hat{u},_{x x}-M_{y} \hat{u},_{y y}-2 M_{x y} \hat{u},_{x y}
\end{aligned}
$$

we obtain the equality

$$
\begin{aligned}
& \delta \theta\left[-M_{x} \hat{u},_{x x}-M_{y} \hat{u},{ }_{y y}-2 M_{x y} \hat{u},_{x y}\right]+\delta \psi\left[-M_{x} \hat{v},_{x x}-M_{y} \hat{v},_{y y}-2 M_{x y} \hat{v},_{x y}\right]= \\
& =\delta \hat{u}\left[\Omega,{ }_{x} \hat{w},{ }_{y}-\Omega,{ }_{y} \hat{w},_{x}\right]+\delta \hat{v}\left[R,{ }_{y} \hat{w},_{x}-R, \hat{w}_{x}\right]+ \\
& +\delta \hat{w}\left[R,{ }_{x}\left(1+\hat{v},{ }_{y}\right)-R,{ }_{y} \hat{v},_{x}+\Omega,_{y}\left(1+\hat{u},{ }_{x}\right)-\Omega,{ }_{x} \hat{u},_{y}\right]+ \\
& +\frac{\partial}{\partial x}\left[-R\left(1+\hat{v},{ }_{y}\right) \delta \hat{w}+R \hat{w},{ }_{y} \delta \hat{v}-\Omega \hat{w},{ }_{y} \delta \hat{u}+\Omega \hat{u},{ }_{y} \delta \hat{w}\right]+ \\
& +\frac{\partial}{\partial y}\left[-R \hat{w},_{x} \delta \hat{v}+R \hat{v}{ }_{x} \delta \hat{w}-\Omega\left(1+\hat{u},{ }_{x}\right) \delta \hat{w}+\Omega \hat{w},_{x} \delta \hat{u}\right]
\end{aligned}
$$


Finally, collecting all the terms in (20)-(25) and accounting (28), we get the variation of the strain potential energy as

$$
\delta \Pi=\int_{\Gamma} d \Gamma\left(\frac{\partial P_{x}}{\partial x}+\frac{\partial P_{y}}{\partial y}+K_{u} \delta \hat{u}+K_{v} \delta \hat{v}+K_{w} \delta \hat{w}\right)
$$

where

$$
\begin{aligned}
& K_{u}=-N_{x, x}-N_{x y, y}-\left(N_{x} \hat{u},_{x}+N_{x y} \hat{u},_{y}\right)_{,_{x}}-\left(N_{y} \hat{u},_{y}+N_{x y} \hat{u},_{x}\right)_{y}-\left(M_{x} \theta\right)_{x x}-\left(M_{y} \theta\right)_{, y y}- \\
& -2\left(M_{x y} \theta\right),_{x y}+\Omega,{ }_{x} \hat{w},{ }_{y}-\Omega,{ }_{y}{ }_{{ }_{x}} \\
& K_{v}=-N_{y, y}-N_{x y, x}-\left(N_{x} \hat{v},_{x}+N_{x y} \hat{v},_{y}\right),_{x}-\left(N_{y} \hat{v},_{y}+N_{x y} \hat{v},_{x}\right),_{y}-\left(M_{x} \psi\right),_{x x}-\left(M_{y} \psi\right)_{y y}- \\
& -2\left(M_{x y} \psi\right)_{x y}+R,{ }_{y}{ }_{x}-R,{ }_{x} \hat{w},_{y} \\
& K_{w}=-M_{x, x x}-M_{y, y y}-2 M_{x y, x y}-\left(N_{x} \hat{w},_{x}+N_{x y} \hat{w},_{y}\right),_{x}-\left(N_{y} \hat{w},_{y}+N_{x y} \hat{w},_{x}\right){ }_{y}+R,,_{x}\left(1+\hat{u},_{x}\right)- \\
& -R,{ }_{y},_{x}+\Omega,{ }_{y}\left(1+\hat{u},{ }_{x}\right)-\Omega,{ }_{x} \hat{u}{ }_{y} \\
& P_{x}=\left\lfloor N_{x}\left(1+\hat{u},_{x}\right)+N_{x y} \hat{u},_{y}+\left(M_{x} \theta\right),_{x}+\left(M_{x y} \theta\right),{ }_{y}-\Omega \hat{w},{ }_{y}\right\rfloor \delta \hat{u}+ \\
& +\left[N_{x y}\left(1+\hat{v},_{y}\right)+N_{x} \hat{v},_{x}+\left(M_{x} \psi\right),_{x}+\left(M_{x y} \psi\right),_{y}+R \hat{w},{ }_{y}\right] \delta \hat{v}+ \\
& +\left[N_{x y} \hat{w},_{y}+N_{x} \hat{w},_{x}+M_{x, x}+M_{x y, y}-R\left(1+\hat{v},_{y}\right)+\Omega \hat{u},{ }_{y}\right] \delta \hat{w}- \\
& -\left(M_{x} \theta\right) \delta \hat{u},{ }_{x}-\left(M_{x} \psi\right) \delta \hat{v},_{x}-M_{x} \delta \hat{w},,_{x}-M_{x y} \theta \delta \hat{u},{ }_{y}-M_{x y} \psi \delta \hat{v},{ }_{y}-M_{x y} \delta \hat{w},_{y} \\
& P_{y}=\left[N_{x y}\left(1+\hat{u},{ }_{x}\right)+N_{y} \hat{u},{ }_{y}+\left(M_{x y} \theta\right),_{x}+\left(M_{y} \theta\right)_{y}+\Omega \hat{w},{ }_{x}\right\rfloor \hat{u}+ \\
& +\left[N_{y}\left(1+\hat{v},_{y}\right)+N_{x y} \hat{v}_{x}+\left(M_{x y} \psi\right),_{x}+\left(M_{y} \psi\right)_{y}-R \hat{w},{ }_{x}\right] \delta \hat{v}+ \\
& +\left[N_{y} \hat{w},_{y}+N_{x y} \hat{w},_{x}+M_{y, y}+M_{x y, x}+R \hat{v},_{x}-\Omega\left(1+u,{ }_{x}\right)\right] \delta \hat{w}- \\
& -M_{x y} \theta \delta \hat{u},_{x}-M_{x y} \psi \delta \hat{v},_{x}-M_{x y} \delta \hat{w},_{x}-M_{y} \theta \delta \hat{u},{ }_{y}-M_{y} \psi \delta \hat{v},_{y}-M_{y} \delta \hat{w},_{y}
\end{aligned}
$$

Now we compare the relations (30)-(34) with the results of (Selyugin 2021). As we see, the obtained expressions (30)-(34) are identical to the ones of the cited paper.

The next step of our analysis is devoted to the potential of external forces.

\subsection{Potential of external forces}

The potential of external forces $W$ is given by the expression:

$$
W=\int_{\Gamma} q(x, y) \hat{w} d \Gamma+\int_{S_{1}}\left[f_{x} u+f_{y} v+f_{z} w\right] d S
$$


The potential is linear wrt to the displacements. It is shown in (Selyugin 2021) that the variation of the last integral in (35) may be transformed as follows:

$$
\begin{aligned}
& \int_{S_{1}}\left[f_{x} \delta u+f_{y} \delta v+f_{z} \delta w\right] d S= \\
& =\int_{C_{1}} d C\left\{F_{x} \delta \hat{u}+F_{y} \delta \hat{v}+F_{z} \delta \hat{w}+\left[\left(-\widetilde{M}_{y} \hat{w},_{y}+\widetilde{F}_{z}\left(1+\hat{v}_{,}\right)\right) l+\left(\widetilde{M}_{y} \hat{w},{ }_{x}-\widetilde{F}_{z} \hat{v},_{x}\right) m\right] \delta \hat{u},_{n}+\right. \\
& +\left[-\left(-\widetilde{M}_{y} \hat{w},_{y}+\widetilde{F}_{z}\left(1+\hat{v}_{y}\right)\right) m+\left(\tilde{M}_{y} \hat{w},_{x}-\widetilde{F}_{z} \hat{v}_{x}\right) l\right] \delta \hat{u},_{s}+ \\
& +\left[\left(\tilde{M}_{x} \hat{w}_{y}-\widetilde{F}_{z} \hat{u},{ }_{y}\right) l+\left(-\tilde{M}_{x} \hat{w},_{x}+\widetilde{F}_{z}\left(1+\hat{u},{ }_{x}\right)\right) m\right] \delta \hat{v}_{,_{n}}+ \\
& +\left[-\left(\widetilde{M}_{x} \hat{w},{ }_{y}-\widetilde{F}_{z} \hat{u},_{y}\right) m+\left(-\widetilde{M}_{x} \hat{w},_{x}+\widetilde{F}_{z}\left(1+\hat{u},_{x}\right)\right)\right] \delta \hat{v},_{s}+ \\
& +\left[\left(-\tilde{M}_{x}\left(1+\hat{v},{ }_{y}\right)+\tilde{M}_{y} \hat{u},{ }_{y}\right) l+\left(\tilde{M}_{x} \hat{v}_{{ }_{x}}-\tilde{M}_{y}\left(1+\hat{u},{ }_{x}\right)\right) m\right] \delta \hat{w}_{,_{n}}+ \\
& \left.+\left[-\left(-\tilde{M}_{x}\left(1+\hat{v},{ }_{y}\right)+\tilde{M}_{y} \hat{u},{ }_{y}\right) m+\left(\tilde{M}_{x} \hat{v}_{x_{x}}-\tilde{M}_{y}\left(1+\hat{u},{ }_{x}\right)\right)\right] \delta \hat{w},_{s}\right\}
\end{aligned}
$$

It is shown in (Selyugin 2021) that in case of valid relation of the virtual work principle

$$
\int_{V} d V\left[\sigma_{x x} \delta \varepsilon_{x x}+\sigma_{y y} \delta \varepsilon_{y y}+2 \sigma_{x y} \delta \varepsilon_{x y}\right]-\int_{\Gamma} q(x, y) \delta w d \Gamma-\int_{S_{1}}\left[f_{x} \delta u+f_{y} \delta v+f_{z} \delta w\right] d S=0
$$

the relation (36) leads to the following boundary conditions:

- $\quad$ at the loaded portion $C_{1}$ of the boundary:

$$
\begin{aligned}
& \mid\left(1+\hat{u},{ }_{x}\right)\left(N_{x} l+N_{x y} m\right)+\hat{u},{ }_{y}\left(N_{x y} l+N_{y} m\right)+\Omega\left(-l \hat{w},_{y}+m \hat{w},_{x}\right)+ \\
& \left.+l\left(\left(M_{x} \theta\right),_{x}+\left(M_{x y} \theta\right),_{y}\right)+m\left(\left(M_{x y} \theta\right),_{x}+\left(M_{y} \theta\right),{ }_{y}\right)\right]+\frac{\partial A_{u}}{\partial s}-F_{x}=0 \\
& \mid \hat{v}_{,}\left(l N_{x}+m N_{x y}\right)+\left(1+\hat{v}_{y}\right)\left(l N_{x y}+m N_{y}\right)+R\left(l \hat{w},{ }_{y}-m \hat{w},_{x}\right)+ \\
& \left.+l\left(\left(M_{x} \psi\right),_{x}+\left(M_{x y} \psi\right),_{y}\right)+m\left(\left(M_{x y} \psi\right),_{x}+\left(M_{y} \psi\right),{ }_{y}\right)\right]+\frac{\partial A_{v}}{\partial s}-F_{y}=0 \\
& {\left[l\left(N_{x} \hat{w},_{x}+N_{x y} \hat{w},_{y}+M_{x, x}+M_{x y, y}-R\left(1+\hat{v},_{y}\right)+\Omega \hat{u},_{y}\right)-\right.} \\
& \left.+m\left(N_{x y} \hat{w},_{x}+N_{y} \hat{w},_{y}+M_{x y, x}+M_{y, y}+R \hat{v},{ }_{x}-\Omega\left(1+\hat{u},{ }_{x}\right)\right)\right]+\frac{\partial A_{w}}{\partial s}-F_{z}=0 \\
& -\left(l^{2} M_{x} \theta+2 \operatorname{lm} M_{x y} \theta+m^{2} M_{y} \theta\right)-\left[\left(-\widetilde{M}_{y} \hat{w},_{y}+\widetilde{F}_{z}\left(1+\hat{v},_{y}\right)\right) l+\left(\widetilde{M}_{y} \hat{w},_{x}-\widetilde{F}_{z} \hat{v}_{x}\right) m\right]=0 \\
& -\left(l^{2} M_{x} \psi+2 \operatorname{lm} M_{x y} \psi+m^{2} M_{y} \psi\right)-\left[\left(\tilde{M}_{x} \hat{w},{ }_{y}-\widetilde{F}_{z} \hat{u},{ }_{y}\right) l+\left(-\tilde{M}_{x} \hat{w},_{x}+\widetilde{F}_{z}\left(1+\hat{u},_{x}\right)\right) m\right]=0 \\
& -\left(l^{2} M_{x}+2 \operatorname{lm} M_{x y}+m^{2} M_{y}\right)-\left[\left(-\tilde{M}_{x}\left(1+\hat{v}_{y}\right)+\tilde{M}_{y} \hat{u},{ }_{y}\right) l+\left(\tilde{M}_{x} \hat{v}_{,_{x}}-\tilde{M}_{y}\left(1+\hat{u},,_{x}\right)\right) m\right]=0
\end{aligned}
$$

- $\quad$ and at $C_{2}$ to the clamping conditions:

$$
\delta \hat{u}=\delta \hat{v}=\delta \hat{w}=\delta \hat{u},_{n}=\delta \hat{v},_{n}=\delta \hat{w},_{n}=0
$$

where

$$
\begin{aligned}
& A_{u}=\left(\operatorname{lm}\left(M_{x}-M_{y}\right) \theta-\left(l^{2}-m^{2}\right) M_{x y} \theta\right)+\left[\left(-\widetilde{M}_{y} \hat{w},_{y}+\widetilde{F}_{z}\left(1+\hat{v},{ }_{y}\right)\right) m-\left(\widetilde{M}_{y} \hat{w}_{x_{x}}-\widetilde{F}_{z} \hat{v}_{x}\right) l\right] \\
& A_{v}=\left(\operatorname{lm}\left(M_{x}-M_{y}\right) \psi-\left(l^{2}-m^{2}\right) M_{x y} \psi\right)+\left[\left(\widetilde{M}_{x} \hat{w}_{,_{y}}-\widetilde{F}_{z} \hat{u}_{y}\right) m-\left(-\tilde{M}_{x} \hat{w}_{x_{x}}+\widetilde{F}_{z}\left(1+\hat{u},_{x}\right)\right)\right] \\
& A_{w}=\left(\operatorname{lm}\left(M_{x}-M_{y}\right)-\left(l^{2}-m^{2}\right) M_{x y}\right)+\left[\left(-\tilde{M}_{x}\left(1+\hat{v}_{, y}\right)+\tilde{M}_{y} \hat{u},_{y}\right) m-\left(\tilde{M}_{x} \hat{v}_{x}-\tilde{M}_{y}\left(1+\hat{u},{ }_{x}\right)\right)\right]
\end{aligned}
$$

The conditions (44) are the essential ones. The conditions (38)-(43) are the natural ones. 
Now we demonstrate that the relations (25)-(47) are a consequence of a kinematic variational principle described in the following sub-Section.

\subsection{A kinematic variational principle}

The principle is formulated as follows:

$$
\Pi-W \underset{\hat{u}, \hat{v}, \hat{w}}{\longrightarrow} \text { stationarity under the conditions (44) }
$$

Indeed, we obtain from (48) the equilibrium equations

$$
\begin{aligned}
& K_{u}=0 \\
& K_{v}=0 \\
& K_{w}-q=0
\end{aligned}
$$

and the proper boundary conditions (38)-(43), (45)-(47).

\section{Discussion}

The proposed variational principle leads to the equilibrium equations. Every equilibrium equation from (49) expressed in $\hat{u}, \hat{v}, \hat{w}$ has the order four. Hence, the order of the equilibrium equation system (49) is twelve. There are six unknown primary variables $\hat{u}, \hat{v}, \hat{w}, \hat{u},_{n}, \hat{v},_{n}, \hat{w},_{n}$ to be determined from (49). We obtain six natural boundary conditions (38)-(43) at $C_{1}$ contour portion, with the essential boundary conditions (44) at $C_{2}$ being prescribed in the principle.

When we consider the moderate deflections only, it is easy to see that in the quantities $K_{u}, K_{v}, K_{w}$ respectively two, two and five initial terms (corresponding to the von Karman case) remain. In the boundary conditions the variables $\theta, \psi$ become equal to zero. The quantities

$A_{u}, A_{v}$ disappear (or, in other words, equal to zero). The quantities $\hat{u},_{x}, \hat{u},{ }_{y}, \hat{v},_{x}, \hat{v},_{y}$ are neglected and taken equal to zero. The conditions (38)-(40), (43) are transformed to the von Karman case (see Reddy 2007). The conditions (41), (42) disappear. The variables $\hat{u}, \hat{v}, \hat{u},_{n}, \hat{v},_{n}$ leave the list of the primary ones. The consideration shows that the proposed principle in case of moderate deflections is valid for the von Karman case.

\section{Conclusions}

- A kinematic variational principle is derived

- There are six primary variables in the system, namely $\hat{u}, \hat{v}, \hat{w}, \hat{u},{ }_{n}, \hat{v},{ }_{n}, \hat{w}{ }_{n}$

- The essential boundary conditions are imposed from the very beginning; the natural boundary conditions follow from the stationarity conditions.

- For moderate deflections the principle is transformed into one of the von Karman case

- The derived principle may be used for numerical solutions of the nonlinear plate static problems. 


\section{REFERENCES}

Gibson R., 1994. Principles of composite material mechanics. McGraw-Hill, 425 pp.

Novozhilov V., 2011. Foundations of the nonlinear theory of elasticity. Dover Publ., 256 pp.

Novozhilov V., 1961. Theory of elasticity. Pergamon Press, 448 pp.

Reddy J.N., 2007. Theory and analysis of elastic plates and shells. $2^{\text {nd }}$ edition. Taylor $\&$ Francis Group, New York.

Selyugin S., 2021. Thin metallic and composite plates experiencing large deflections above the von Karman limits. https://doi.org/10.31224/osf.io/hr79j

Washizu K., 1975. Variational Methods in Elasticity and Plasticity. $2^{\text {nd }}$ edition, Pergamon Press. $412 \mathrm{pp}$. 